\title{
Which Non-Muscle Invasive Bladder Cancer Is More Valuable For Re-transurethral resection?
}

\author{
Murat Akgul ${ }^{1}$, Cagri Dogan ${ }^{2}$, Cenk Murat Yazıcı ${ }^{1}$, Mehmet ŞAHIN² ${ }^{2}$ Aysegul Arslan², and \\ Meltem Oznur ${ }^{1}$ \\ ${ }^{1}$ Namik Kemal University Faculty of Medicine \\ ${ }^{2}$ Tekirdag Namik Kemal University
}

April 10, 2021

\begin{abstract}
INTRODUCTION: We evaluated the re-transurethral resection (re-TUR) pathologies and the comparison of pathology results between transurethral resection of bladder (TUR-B) and re-TUR for non-muscle invasive bladder cancer (NMIBC). Additionally we assessed the factors affecting the re-TUR pathology and tried to define the more valuable re-TUR patient groups. We also aimed to evaluate the effect of re-TUR on recurrence and progression. METHODS: We performed re-TUR to intermediate/high risk NMIBC patients, 4-6 weeks after the index TUR-B. Both TUR-B and re-TUR pathology characteristics, including tumor stage, grade, size, number, lymphovascular invasion (LVI), carcinoma in situ (CIS), variant pathology and intermediate/high risk status were analysed. The recurrence and progression rates were also evaluated according to re-TUR. RESULTS: A total of 78 patients with NMIBC were included to the study. The index TUR-B pathologies were Ta-Low: 6 (7,7\%), Ta-High: 5 (6,4\%), T1-Low: 14 (17,9\%), T1-High: 53 (67,9\%). Re-TUR positivity was n: 40 (51\%), and upstaging/upgrading at re-TUR was n: $11(14 \%)$ in all groups. Re-TUR positivity was significantly higher in high-risk compared to intermediate-risk NMIBC (p:0,026). Re-TUR positivity was higher in patients with hydronephrosis, CIS, LVI, differentiation, size $(>3 \mathrm{~cm})$ and multiple tumour presence $(\mathrm{p}<0,05)$. There was no significant relationship between recurrence / progression and re-TUR ( $>0,05)$. CONCLUSION: Residual tumour was common after the index TUR-B and upstaging after re-TUR was very important. ReTUR is critically important in high-risk NMIBC, presence of hydronephrosis, CIS, LVI, variant pathology, size $(>3 \mathrm{~cm})$ and multiple number of tumor.
\end{abstract}

Which Non-Muscle Invasive Bladder Cancer Is More Valuable For Re-transurethral resection? ABSTRACT

INTRODUCTION: We evaluated the re-transurethral resection (re-TUR) pathologies and the comparison of pathology results between transurethral resection of bladder (TUR-B) and re-TUR for non-muscle invasive bladder cancer (NMIBC). Additionally we assessed the factors affecting the re-TUR pathology and tried to define the more valuable re-TUR patient groups. We also aimed to evaluate the effect of re-TUR on recurrence and progression.

METHODS: We performed re-TUR to intermediate/high risk NMIBC patients, 4-6 weeks after the index TUR-B. Both TUR-B and re-TUR pathology characteristics, including tumor stage, grade, size, number, lymphovascular invasion (LVI), carcinoma in situ (CIS), variant pathology and intermediate/high risk status were analysed. The recurrence and progression rates were also evaluated according to re-TUR.

RESULTS: A total of 78 patients with NMIBC were included to the study. The index TUR-B pathologies were Ta-Low: 6 (7,7\%), Ta-High: 5 (6,4\%), T1-Low: 14 (17,9\%), T1-High: 53 (67,9\%). Re-TUR positivity was n: 40 (51\%), and upstaging/upgrading at re-TUR was n: 11 (14\%) in all groups. Re-TUR positivity 
was significantly higher in high-risk compared to intermediate-risk NMIBC (p:0,026). Re-TUR positivity was higher in patients with hydronephrosis, CIS, LVI, differentiation, size $(>3 \mathrm{~cm})$ and multiple tumour presence $(\mathrm{p}<0,05)$. There was no significant relationship between recurrence / progression and re-TUR $(\mathrm{p}>0,05)$.

CONCLUSION: Residual tumour was common after the index TUR-B and upstaging after re-TUR was very important. Re-TUR is critically important in high-risk NMIBC, presence of hydronephrosis, CIS, LVI, variant pathology, size $(>3 \mathrm{~cm})$ and multiple number of tumor.

Keywords: Bladder Cancer, Tur-B, Re-Tur, Pathology

\section{WHAT IS ALREADY KNOWN ABOUT THIS TOPIC?}

Re-transurethral resection (Re-TUR) surgery is recommended to prevent misclassification or to resect invisible residual tumors after the index transurethral resection of bladder (TUR-B) according to index pathology. If there is no muscle tissue in pathology specimen or the index TUR-B is incomplete, complementary TUR-B should be performed. However, Re-TUR is a totally different procedure from complementary TURB. Re-TUR is performed after complete TUR-B. The necessity of re-TUR was not uniformly accepted and there is a significant gap in the literature for choosing the optimal re-TUR patients.

\section{WHAT DOES THIS ARTICLE ADD?}

The necessity of Re-TUR surgery is questioned in patients with comorbidities, frail elderly patients and difficult periods such as COVID-19 pandemics. The results showed that Re-TUR is especially important in patients with high-risk non-muscle invasive bladder cancer, presence of hydronephrosis, carcinoma in situ, lymphovascular invasion, variant pathology, size $(3 \mathrm{~cm}>)$ and multiple number of tumors.

\section{INTRODUCTION}

Bladder cancer (BC) is the 7th most common cancer in males and 13th most mortal cancer in both males and females ${ }^{1}$. Smoking, genetic factors, chemical agents and many other factors are the risk factors for the aetiology of $\mathrm{BC}^{2-4}$. Approximately $75 \%$ of $\mathrm{BC}$ is non-muscle invasive bladder cancer (NMIBC) and $25 \%$ is muscle invasive bladder cancer (MIBC) at the time of diagnosis ${ }^{5}$. Differentiating the NMIBC from MIBC is so important because the treatment protocol is totally different. If the patients were misdiagnosed as NMIBC instead of MIBC, it would be catastrophic for the treatment strategy. To prevent this situation, re-TUR (retransurethral resection) is performed 2-6 weeks after the index TUR-B (transurethral resection of bladder) operation $^{6}$. Complementary TUR-B should be performed, if there is no muscle tissue at pathology specimen or the index TUR-B is incomplete. However re-TUR is a totally different procedure from complementary TUR-B. Re-TUR is performed after complete TUR-B to prevent misclassification or to resect undetected tumors after the index TUR-B ${ }^{7}$.

The European Urology Association (EAU) guidelines defined Ta_Low grade tumors as low-risk NMIBC category, which means that the risk of progression is low for this group. On the other hand, T1 tumors, high-grade tumors, carcinoma in situ (CIS) pathologies and all features including multiple, recurrent and large $(>3 \mathrm{~cm})$ tumors are in the high-risk NMIBC group. The rate of progression in this group is significantly higher than low-risk group ${ }^{8-9}$. Pathologies between these two groups are considered to be intermediate-risk group for NMIBC. Re-TUR is proposed to be unnecessary in low-risk NMIBC. On the other hand, in highrisk NMIBC, re-TUR is routinely recommended. However there is no exact recommendation about the feasibility of re-TUR for intermediate risk $\mathrm{NMIBC}^{9}$.

In this study, we aimed to evaluate the re-TUR pathologies and the comparison of pathology results between TUR-B and re-TUR for intermediate and high-risk NMIBC. In addition we aimed to evaluate the more valuable patient groups for re-TUR and factors affecting re-TUR pathology. We also aimed to assess the influence of re-TUR on recurrence and progression for intermediate and high-risk NMIBC patients.

\section{METHODS}

\section{Study population and protocol}


With the permission of the local ethics committee, the patients to whom re-TUR operation was performed between 2013-2020 in our clinic were retrospectively included to the study. Patients underwent TUR-B operation under general or spinal anaesthesia by the continuous flow 27 French $30^{\circ}$ optic resectoscope instrument and video camera system (Karl StorzTM, Tuttlingen, Germany).

The patients who were at intermediate and high-risk classification were included to the study. The patients with Ta_Low grade pathology that present one of these parameters; multiple, recurrent or $>3 \mathrm{~cm}$ tumors were considered as intermediate risk NMIBC. Therefore re-TUR was also applied for these Ta_Low grade patients who were classified in the intermediate risk group. On the other hand, the patients who had low-risk NMIBC, patients with MIBC, patients with in-complete TUR-B and the patients who had no muscle tissue in the pathologic specimen, were excluded from the study.

The re-TUR operation was performed approximately 4-6 weeks after the index TUR-B for intermediate and high-risk NMIBC patients. During re-TUR operation, resection was performed from the same area of the primary tumor including the deep muscle layer, regardless of the residual or recurrent tumour. The re-TUR specimen was checked for any residual tumor and whether there was a change in tumour stage or grade. All the patients who were not upstaged to MIBC received standard BCG immunotherapy.

Both TUR-B and re-TUR pathology characteristics including tumor stage, tumor grade, tumor size, tumor number, presence of lymphovascular invasion (LVI), presence of CIS, presence of variant pathology and the tumor risk status were analysed. The Re-TUR positivity (residual tumor) of the patients were evaluated and analysed according to these variables.

\section{Statistical Analysis}

In descriptive statistics of the data; frequency, ratio, mean and standard deviation values were used. Continuous data are reported as means \pm standard deviations (SD) or median values as appropriate. Statistical analyses were done with the SPSS 21.0 package program. Statistical analyses were carried out with the chi-square / Fisher's Exact test in cross tables. P $<0.05$ was considered statistically significant.

\section{RESULTS}

A total of 78 patients with intermediate and high risk NMIBC were included to the study. The mean age of the patients was $63.9 \pm 9.0$ (38- 85 years). There were $72(92,3 \%)$ male and $6(7,7 \%)$ female patients. The mean follow-up time was 42,1 $\pm 29,6$ (min 10- max 142) months. According to EAU risk classification; 6 (7,7 $\%)$ patients were in the intermediate risk group and $72(92,3 \%)$ patients were in the high-risk group. The index TUR-B and re-TUR pathologies of the patients were shown in Table 1.

Most of the re-TUR positivity was seen in high_grade patients. Three of (60\%) Ta_High grade patients (n:5) had re-TUR positivity and 1 of them upstaged to MIBC. T1_High grade patients (n:53) had the highest number of re-TUR positivity (n:31) (58,5\%). Eight of the $\overline{\mathrm{T}} 1$ _High grade patients upstaged to MIBC (15\%). The patients to whom upstaged to T2 treated with 'radical cystectomy' or 'radiotherapy + chemotherapy'. On the other hand none of the Ta_Low grade patients (n:6) had re-TUR positivity (Table 1).

Re-TUR positivity and up-stage/grade was demonstrated according to tumor stage, tumour grade and intermediate / high-risk NMIBC in Table 2. Re-TUR positivity was n: 40 (51 \%), and upstaging/upgrading at re-TUR was n: $11(14 \%)$ in all groups. There was a statistical significant difference between Re-TUR positivity and T stage/grade (p:0.031). In addition, re-TUR positivity was significantly higher in high-risk compared to intermediate-risk NMIBC (p:0.026). Although the recurrence (n:18) and progression (n:11) were higher in the high_risk group (n:72), the statistical analysis did not show any significant difference ( $p>0.05)$. The odds ratio of re-TUR positivity was 5,57 for recurrence $(95 \% \mathrm{CI}: 1,7-18,5)$ and 6,52 for progression (95\% CI: 1,3-33,5).

Re-TUR positivity was significantly higher in patients with hydronephrosis, CIS, LVI, variant pathology, size $(3 \mathrm{~cm}>)$ and multiple tumour presence $(\mathrm{p}<0.05)$ (Table 3$)$. In addition different tumor variants such as sarcomatoid, neuroendocrine, micropapillary, plasmacytoid differentiation had worse prognosis than others. In 
our study there were 4 patients with different variants and 3 of them $(75 \%)$ had re-TUR positivity.

\section{DISCUSSION}

Bladder cancer is an aggressive tumor with high morbidity and mortality rate. It is really important to choose the best treatment option for BC. The EAU guidelines recommend re-TUR for possible upstaging of NMIBC to invasive cancer and for clearance of residual tumor after index TUR-B ${ }^{9}$. It was demonstrated that the presence of residue and tumour upstaging were high in re-TUR series. Therefore re-TUR is critically important for complete resection and re-staging after index TUR. Disease management and mortality of the patients may totally change by the help of re-TUR pathology. Although there are different recommendations for the timing of re-TUR, the most accepted time of the procedure is 2 to 6 weeks after the index TUR- ${ }^{10}$.

The upstaging rates of T1 patients were found to be high in the literature. Fritsche et al analysed the data of 1136 patients treated with radical cystectomy for clinical T1 high stage group and demonstrated that nearly half of the pT1 patients $(49,7 \%)$ had MIBC pathology ${ }^{11}$. These rates supported the inadequacy of clinical decision-making based on current treatment paradigms and staging tools for T1_high stage tumors. Herr et al also stated that re-TUR revealed up to $43 \%$ upstaging and up to $85 \%$ of re-TUR positivity ${ }^{12}$. The re-TUR positivity was $51 \%$ and upstaging rate was $14 \%$ in our study. In recently published reviews, the residual tumor rates were approximately $51-58 \%$ and T2 upstaging rates were 8-11\%, which were similar to our study results ${ }^{13-14}$. The subgroup analysis in the literature documented that re-TUR positivity was 17-67 $\%$ in Ta patients and $20-71 \%$ in T1 patients. Most residual tumours (36-86 \%) were found at the original resection site ${ }^{14}$. In our study, the re-TUR positivity was found $27 \%$ for Ta and $55 \%$ for T1 patients that were similar to literature.

The necessity of re-TUR was not uniformly accepted. Some authors did not recommend re-TUR due to the low percentage of upstaging, possible complications and the cost of the surgery ${ }^{15}$. Gaya et al claimed that re-TUR is mandatory only if there was no muscle tissue in the initial resection specimen. They thought that absence of muscle is the only risk factor for understaging ${ }^{16}$. However their patient population was low and the lack of muscle tissue is actually the reason for complementary TUR-B not for re-TUR. In our study, the re-TUR positivity of T1_high grade patients was $58 \%$ and upstaging rate was $15 \%$. T1 high grade is the highest stage for NMIBC and these patients are at the edge of the border for MIBC. Therefore it would be proper to make re-TUR to these patients. On the other hand, the re-TUR is an invasive operation; the pros and cons of the procedure also should be considered. There are also question marks about the necessity of re-TUR especially in the COVID-19 pandemic period. The clinicians must be more careful to make the surgical indications for their patients in terms of both patient and public health ${ }^{17}$. Any surgical procedure that does not have significant indication must be questioned. Shared decision making would be a solution for this situation. By this way, clinicians should discuss the decision of re-TUR requirement collectively with the patients in the light of evidence-based literature.

The impact of re-TUR on the long-term outcomes for T1 patients was discussed in several studies in literature $^{16-20}$. Divrik et al revealed in their prospective randomised clinical trial that re-TUR had significantly decreased the recurrence and progression rates in patients with newly diagnosed $\mathrm{T} 1$ stage $^{18}$. In addition to this Sfakianos et al claimed that the absence of re-TUR before initiating intravesical BCG therapy for high risk NMIBC significantly increased the risk of recurrence ${ }^{19}$. On the other hand, some studies claimed that the recurrence and progression of T1_high grade patients treated with BCG without re-TUR was not as bad as previously thought ${ }^{20}$. They reported that the oncological results and the rate of recurrence / progression will not be affected after re-TUR for T1_high-grade. Moreover, re-TUR can cause patient distress and higher re-operation related healthcare $\operatorname{costs}^{21}$. In our study, we could not find any statically significant relation between re-TUR and recurrence/progression. It might be due to appropriate intravesical BCG and other curative treatments plus close follow-up.

The presence of hydronephrosis, CIS, LVI, variant pathology and tumor size $>3 \mathrm{~cm}$ in index TUR are generally associated with a poor prognosis of BC. Bishr et al reported that the adverse prognostic features related with re-TUR were; number of tumours ( $>3$ lesions), tumour size $(>3 \mathrm{~cm})$, hydronephrosis, invasion of the lamina 
propria (T1), high-grade, concomitant CIS, different tumour variants and T1 stage ${ }^{22}$. We also analysed these variables in terms of re-TUR positivity. We examined that the re-TUR positivity rate was significantly higher in patients with these variables. When these factors present, it would be critically important to perform re-TUR without a doubt.

\section{Limitations}

The retrospective study design was one of our limitations. However, according to our clinical policy, we performed strict follow-up protocol for BC patients. The strict follow-up protocol of the patients might reduce the potential bias associated with the retrospective nature of the study. The relatively low number of patients was another limitation of our study. This might be related with the exclusion criteria of our study population. In order to standardize our study population, patients with incomplete index TUR-B and patients with no muscle tissue at index TUR-B specimen were excluded from the study. Another limitation of our study was the absence of a control group to whom we did not perform re-TUR. However the presence of a control group in high_risk group is an ethical problem for this study design. In our clinical practice, we routinely perform re-TÜR to the high_risk NMIBC patients.

\section{CONCLUSIONS}

Residual tumour is common after index TUR-B. The re-TUR is definitely required to detect this residual tumour and to reveal the cancer upstaging. It should be noted that re-TUR is critically important in highrisk NMIBC, presence of hydronephrosis, CIS, LVI, variant pathology, size $(3 \mathrm{~cm}>)$ and multiple number of tumour. However shared decision-making would be useful in determining the re-TUR indication for selected patients. The effect of re-TUR on recurrence and progression in NMIBC is still a dilemma.

\section{REFERENCES}

1. Antoni S, Ferlay J, Soerjomataram I, et al. Bladder cancer incidence and mortality: A global overview and recent trends.Eur Urol. 2017;71: 96.

2. Miyazaki J, Nishiyama H. Epidemiology of urothelial carcinoma. Int J Urol. 2017;24:730-34.

3. Baykan O, Akgul M, Uren N, et al. The Relationship Between Urothelial Type Bladder Cancer, Plasma 25-Hydroxyvitamin D Levels, and Vitamin D Receptor ApaI BsmI FokI, and TaqI Polymorphisms. Clin Lab .2019,65.10.7754/Clin.Lab.2018.180339.

4. Cumberbatch MGK, Jubber I, Black PC, et al. Epidemiology of Bladder Cancer: A Systematic Review and Contemporary Update of Risk Factors in. 2018. Eur Urol. 2018;74: 784-95.

5. TNM classification of malignant tumors. UICC International Union Against Cancer. 8th edn. Brierley JD, Gospodarowicz M, Wittekind C (Eds. 2017, Wiley-Blackwell)

6. Mostafid AH. Time to re-evaluate and refine re-transurethral resection in bladder cancer?.BJU international . 2016;118:9-10.

7. Adiyat KT, Katkoori D, Soloway CT, De Los Santos R, Manoharan M, Soloway MS. "Complete transurethral resection of bladder tumor" are the guidelines being followed? Urology. 2010;75:36567.

8. Witjes JA, Moonen PMJ, Van Der Heijden AG. Review pathology in a diagnostic bladder cancer trial: effect of patient risk category. Urology. 2006; 67:751-55.

9. Babjuk M, Burger M, Comperat E, et al. EAU guidelines on non-muscle-invasive (Ta, T1 and CIS) bladder cancer, in EAU guidelines. 2020, European Association of Urology guidelines office Arnhem, the Netherlands.

10. Baltacı S, Bozlu M, Yıldırım A, et al. Significance of the interval between first and second transurethral resection on recurrence and progression rates in patients with high-risk non-muscle-invasive bladder cancer treated with maintenance intravesical Bacillus Calmette-Guerin. BJU Int. 116(5), 721-726.

11. Fritsche HM, Burger M, Svatek RS, et al. Characteristics and out- comes of patients with clinical T1 grade 3 urothelial carcinoma treated with radical cystectomy: results from an international cohort. Eur Urol. 2010;57:300

12. Herr HW, Donat SM. A re-staging transurethral resection predicts early progression of superficial 
bladder cancer. BJU Int. 2006;97: 1194:8.

13. Naselli A, Hurle R, Paparella S, et al. Role of Restaging Transurethral Resection for T1 Non-muscle invasive Bladder Cancer: A Systematic Review and Meta-analysis. Eur Urol Focus.2018;4:558-67.

14. Cumberbatch MGK, Foerster B, Catto JWF, et al. Repeat Transurethral Resection in Non-muscleinvasive Bladder Cancer: A Systematic Review. Eur Urol. 2018;73: 925-33.

15. Palou J, Pisano F, Sylvester R, Joniau S, Serretta V, Larré S. Recurrence, progression and cancerspecific mortality according to stage at re-TUR in T1G3 bladder cancer patients treated with BCG: not as bad as previously thought. World journal of urology. 2018;36:1621-27.

16. Gaya JM, Palou J, Cosentino M, Patiño D, Rodríguez-Faba O, Villavicencio H. A second transurethral resection could be not necessary in all high grade non-muscle-invasive bladder tumours. Actas Urológicas Españolas (English). 2012:539-44.

17. Ho HC, Hughes T, Bozlu M, Kadığlu A, Somani BK. What do urologists need to know: Diagnosis, treatment, and follow-up during COVID-19 pandemic. Turkish Journal of Urology. 2020;46:169.

18. Divrik RT, Sahin AF, Yildirim U, Altok M, Zorlu F: Impact of routine second transurethral resection on the long-term outcome of patients with newly diagnosed pT1 urothelial carcinoma with respect to recurrence, progression rate, and disease-specific survival: a prospective randomised clinical trial. Eur Urol.2010;58:185-90.

19. Sfakianos JP, Kim PH, Hakimi AA, Herr HW. The effect of restaging transurethral resection on recurrence and progression rates in patients with nonmuscle invasive bladder cancer treated with intravesical bacillus Calmette-Guerin. J Urol. 2014;191: 341-5.

20. Gontero P, Sylvester R, Pisano F, et al. The impact of re-transurethral resection on clinical outcomes in a large multicentre cohort of patients with T1 high-grade/Grade 3 bladder cancer treated with bacille Calmette-Guérin. BJU international.2016;118:44-52.

21. Calò B, Chirico M, Fortunato F, et al. Is repeat transurethral resection always needed in high-grade T1 bladder cancer?. Frontiers in Oncology. 2019;9.

22. Bishr M, Lattouf JB, Latour M, Saad F. Tumour stage on re-staging transurethral resection predicts recurrence and progression-free survival of patients with high-risk non-muscle invasive bladder cancer. Can Urol Assoc J. 2014;8:306-10.

23. Witjes J, Bruins HM, Cathomas R, et al. EAU Guidelines on Muscle-invasive and Metastatic Bladder Cancer, in EAU Guidelines. 2020, European Association of Urology Guidelines Office Arnhem, The Netherlands.

\section{Hosted file}

TABLE 1.pdf available at https://authorea.com/users/353513/articles/517449-which-non-muscleinvasive-bladder-cancer-is-more-valuable-for-re-transurethral-resection

\section{Hosted file}

TABLE 2.pdf available at https://authorea.com/users/353513/articles/517449-which-non-muscleinvasive-bladder-cancer-is-more-valuable-for-re-transurethral-resection

\section{Hosted file}

TABLE 3.pdf available at https://authorea.com/users/353513/articles/517449-which-non-muscleinvasive-bladder-cancer-is-more-valuable-for-re-transurethral-resection 